\title{
ADVANCED INDUCTION MOTOR ENDERING DESIGN FEATURES FOR HIGH SPEED APPLICATIONS
}

\author{
By: \\ Matthew Caprio \\ Vasileios Lelos \\ John Herbst \\ Jim UpShaw
}

PR 436

Center for Electromechanics

The University of Texas at Austin

PRC, Mail Code R7000

Austin, TX 78712

(512) $471-4496$

$07 / 11 / 2006$ 


\title{
Advanced Induction Motor Endring Design Features for High Speed Applications
}

\author{
Matthew Caprio, Vasileios Lelos, John Herbst, Jim Upshaw \\ Center for Electromechanics \\ The University of Texas at Austin \\ 1 University Station, R7000 \\ Austin, Texas 78712 USA \\ mcaprio@mail.utexas.edu
}

\begin{abstract}
This paper presents advancements in induction motor endring design to overcome mechanical limitations and extend the operating speed range and joint reliability of induction machines. A novel end ring design met the challenging mechanical requirements of this high speed, high temperature, power dense application, without compromising electrical performance. Analysis is presented of the advanced endring design features including a non uniform cross section, hoop stress relief cuts, and an integrated joint boss, which reduced critical stress concentrations, allowing operation under a broad speed and temperature design range. A generalized treatment of this design approach is presented comparing the concept results to conventional design techniques. Additionally, a low temperature joining process of the bar/end ring connection is discussed that provides the required joint strength without compromising the mechanical strength of the age hardened parent metals. A description of a prototype $2 \mathrm{MW}$, $15,000 \mathrm{rpm}$ flywheel motor generator embodying this technology is presented.
\end{abstract}

\section{INTRODUCTION}

Applications increasingly demand higher speed operation from motors, generators, and actuators, in the interest of increasing mechanical power output from compact devices (higher power density), particularly in mobile environments such as automotive, locomotive, and aerospace. A well known limitation of squirrel cage induction machines -- the strength and integrity of the endring to bar joint [1-4] -- has presented an upper bound to their design speed range, often resulting in the selection of switched reluctance or permanent magnet synchronous machines for the highest speed applications.

Recent advancements in induction motor endring design [5] have reduced the loading and stress in the endring and bar joint area, thereby allowing dramatic improvements in operational speed and resulting power density. These endring design features extend the design speed range of induction motors substantially, making the squirrel cage induction machine topology a suitable option for high speed applications. In addition, these design features are also valuable in high operating temperature designs (in which relative growth between the core and endring results in stresses similar to high speed cases) or for improving robustness of joints in conventional low speed applications requiring improved reliability.

This material is based upon work supported by Federal Railroad Administration cooperative agreement, DTFR53-99-H-00006 Modification 5. Any opinions, findings, and conclusions or recommendations expressed in this publication are those of the authors and do not necessarily reflect the view of the Federal Railroad Administration and/or U.S. DOT.
As a general rule, peak output torque of an electrical machine is governed by geometric and material parameters - for a given surface area of steel operating near magnetic saturation, a theoretical limit of output torque results, independent of design speed. The designer seeking higher power has two options: 1) increase the volume of the machine to increase torque (and power proportionally) while maintaining design speed, or 2) increase the design speed while holding volume constant, as the product of speed and torque results in proportionally higher power. Option 1 implies that the higher power machine becomes physically larger and heavier, whereas option 2 results in a higher power machine with no size increase. This trade off is the impetus for higher speed machines in applications demanding lightweight, compact packaging of motors and generators.

\section{ADVANCED ENDRING DESIGN FEATURES}

The motivating application for the endring design advancements discussed in this paper is a motor generator direct-coupled to a large energy storage flywheel for a hybrid electric locomotive propulsion system [6,7]. The continuous duty power rating of the motor generator is $2 \mathrm{MW}$, over a design speed range of 7,500 - 15,000 rpm (selected to provide the required deliverable energy rating of $100 \mathrm{~kW}-\mathrm{hr}$ from the flywheel energy store). Installed length and weight limitations in this mobile environment provided the motivation for the compact design.

In a conventional induction motor rotor design, the end ring is an annular ring supported by the conductors. At high operating speeds and temperatures, the centrifugal and thermal growth of the non-self supported end ring would result in high stresses at the conductors, laminations, and the conductor joint. This configuration also poses a risk of significant mass imbalance due to radial displacement of the unsupported endring. The $290 \mathrm{~m} / \mathrm{s}$ tip speed of this specific application precludes the use of the conventional fabricated endring design.

A combination of advanced endring design features were developed to act in concert to alleviate the strength limitations of the endring-to-bar joint area in the squirrel cage rotor assembly for this high-speed application, seen in Fig. 1. Note: the features described in this paper may be covered under US Provisional Patent Application \#60/647,898. 


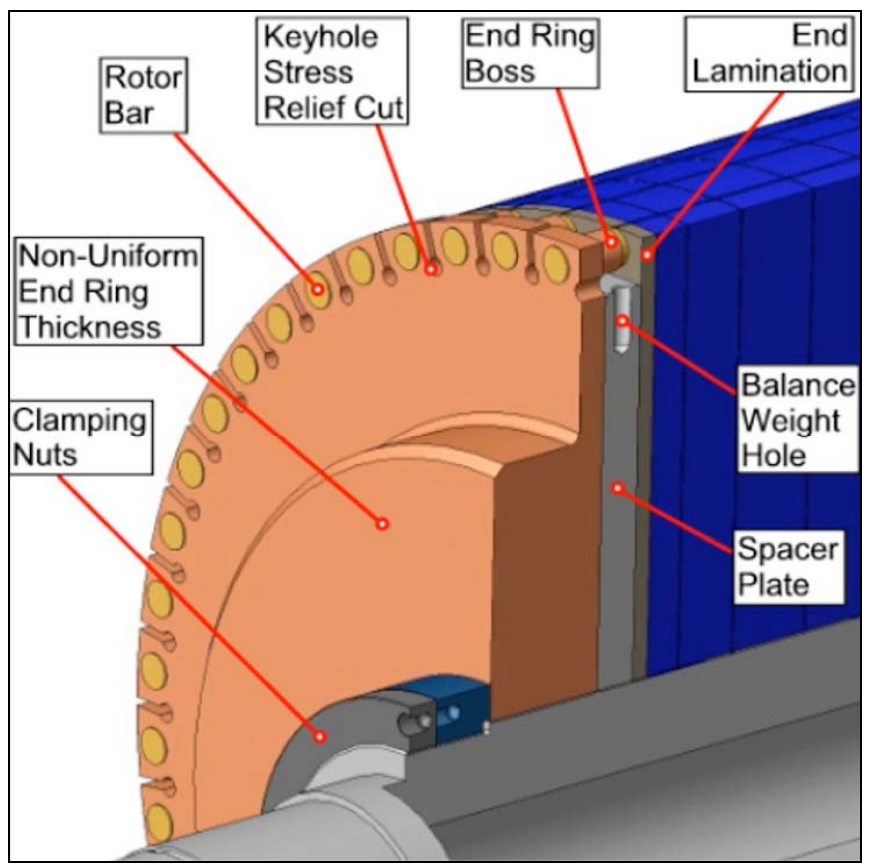

Fig. 1. Section view of advanced endring features

\section{A. Non-Uniform Endring Cross Section}

In this design, the endring is piloted directly to the shaft through an interference fit for rigid support of the endring to ensure that forces associated with imbalance are not transmitted to the rotor bars. However, at these tip speeds, a uniform cross section end ring is not feasible due to separation of the ring from the shaft resulting from the high centrifugal loads. The end ring was therefore designed with a heavier inner diameter section and a thin web extending to the bar radius. This design maintains compressive interface pressure between the shaft and the end ring throughout the speed and temperature ranges of the machine $(0-15,000 \mathrm{rpm}$, -18 to $180^{\circ} \mathrm{C}$ ), with a minimal interference fit that results in manageable stresses.

The thick section endring with direct connection to the shaft serves a secondary purpose of providing bolster support to the lamination stack to prevent conical buckling of the highly interference fitted core necessary for high speed use.

\section{B. Hoop Stress Relief Feature}

Centrifugal loading results in hoop (circumferential) stresses throughout the endring. In a conventional design, these stresses are transmitted through the bar-endring solder joint. For high tip speed applications, the circumferential spin stresses alone can exceed the strength of the solder joint, even without the addition of other contributing loads. In order to isolate the solder joint area from the hoop stresses associated with high speed, a radial stress relief feature was inserted reducing the hoop stresses on the solder joint by a factor of three.

\section{Integral Endring Boss}

A large difference in radial growth exists between the core and the endrings due to centrifugal and thermal loads as the material properties of the core steel and endring copper alloy vary significantly. The radial growth difference naturally exerts a shear load on the rotor bars which connect the two components. To alleviate this effect, the end ring is spaced axially from the laminated core to provide a length over which the relative growth difference of the core and endring can be converted to benign bending. Nevertheless, a stress concentration arises at the joint of the bar and endring which could result in solder joint failure. To gradually distribute this peak contact stress, an integral boss feature is incorporated into the end ring. This feature has the added benefit of increasing the contact area for solder strength and electrical conductivity.

\section{ENDRING MATERIAL SELECTION}

Selecting a material for the endring that balanced the electrical and mechanical material requirements was a challenge in this application. Conventional endring construction die-cast aluminum and fabricated ETP copper materials [8] were considered, but found to be insufficient in strength for this application. Promising recent developments in the use of die-cast copper alloy rotors for high efficiency were reviewed $[9,10]$, but still lack the mechanical strength afforded by fabrication with heat treated materials. Beryllium Copper was selected for adequate strength to withstand the heavy interference fit required to maintain radial contact at the shaft interface during operation at the design speed. Specifically, the selected $\mathrm{BeCu}$ C17510 TH04 material provided the best balance between electrical and mechanical requirements. At this heat treat condition, the material has 63\% IACS conductivity with $97 \mathrm{ksi}$ yield and 102 ksi ultimate strength.

The design implications of choosing a material to satisfy the high mechanical strength and high electrical conductivity requirements resulted in a material with a higher thermal expansion coefficient and lower specific stiffness (Young's modulus divided by the density) than the steel core and shaft. These characteristics resulted in significant thermal and centrifugal radial growth in the end ring. Therefore, a large interference fit was required between the end ring and the shaft to maintain piloting and transmit torque during the machine's wide temperature and speed operating range. The interference fit results in large tangential (hoop) stresses in endring, which are not compatible with most electrical grade materials. Conventional aluminum and electrical coppers, even when heat treated, do not retain adequate mechanical strength after long duration of heat exposure.

A comparison of necessary interference fits and resulting stresses for the candidate materials in a generic uniform cross section endring configuration are summarized in Table 1. 
TABLE I, ENDRING INTERFERENCE AND STRESS FOR CANDIDATE MATERIALS

\begin{tabular}{|l|c|c|c|}
\hline & $\mathrm{Al}$ & $\mathrm{Cu}$ & $\mathrm{BeCu}$ \\
\hline Required Radial Interference (psi) & 0.0085 & 0.0125 & 0.0108 \\
\hline Contact Pressure @ rest (psi) & -20900 & -44700 & -42700 \\
\hline Contact Pressure @ Operation (psi) & 530 & 206 & 157 \\
\hline Ring ID Hoop Stress @ rest (psi) & 25000 & 52700 & 50400 \\
\hline Ring ID Hoop Stress @ Operation (psi) & 30500 & 94700 & 95100 \\
\hline Ring OD Radial Growth @ Operation (in) & 0.0229 & 0.0242 & 0.0225 \\
\hline Typical Yield Strength at Temperature (psi) & 20000 & 40000 & 120000 \\
\hline
\end{tabular}

\section{DESIGN ANALYSIS}

Detailed finite element analysis was required for all regions of the rotor endring as full advantage of these high performance materials was expected in this demanding application.

\section{A. Non-Uniform Endring Cross Section}

An axisymmetric model (Fig. 2) was used to analyze the contact pressure between the interference fitted endring and shaft due to the spin and thermal loading. Analysis demonstrated that the fit resulted in an average residual contact pressure of $10 \mathrm{ksi}$ during a 15,400 rpm overspeed with thermal input. Residual contact pressure on this order is required to transmit torque through the frictional interface in high speed designs due to the unacceptable stress concentrations of conventional keys or spider shafts. The design challenge requires striking the appropriate balance between two conditions: 1) radial contact pressure at speed and elevated temperature to maintain contact, and 2) combined hoop and compressive stresses in the endring at rest, cold to prevent yielding. The analysis results showed this to be possible with the materials and design selected for this endring.

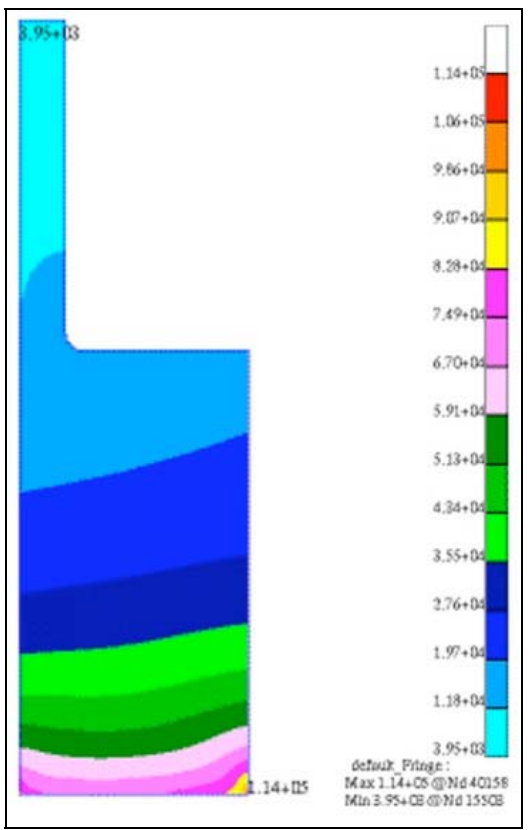

Fig. 2. Stress results of endring interference fit model

\section{B. Hoop Stress Relief Feature}

The use of heat treated materials for the endring and rotor bars applied significant constraints to the allowable time/temperature profiles of these components during the brazing/soldering operations. These limitations required the use of lower temperature (and lower strength) processes for the bar-endring joints. Detailed 3-d FEA was employed to demonstrate that the end ring joint stress relief design features isolate the brazed or soldered bar joint region from the high hoop stresses that accompany high speed and/or high temperature operation (Fig. 3). Isolating the joint from the hoop stress dramatically reduces the load carried by the brazing or soldering filler metal (from $30 \mathrm{ksi}$ to $10 \mathrm{ksi}$ in this case). This feature allows the use of a moderate strength ( 40 ksi) lower temperature $\left(280^{\circ} \mathrm{C}\right)$ alloy such as $80 \mathrm{Au} 20 \mathrm{Sn}$, which can be processed without affecting the heat treated strength of the $\mathrm{BeCu}$, while proving adequate joint strength.
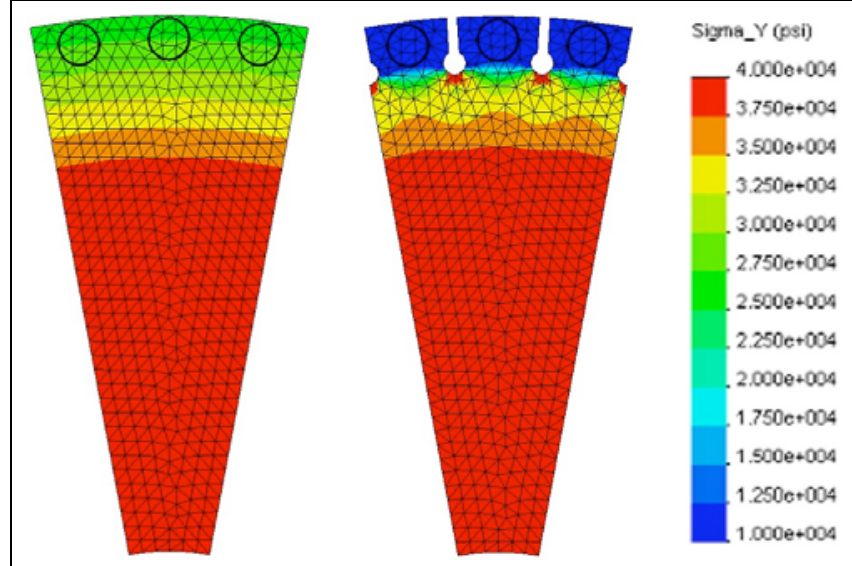

Fig. 3. Comparison of predicted hoop stresses with and without relief feature

\section{Integral Endring Boss}

Detailed analysis of the joint area required coupled thermal-mechanical 3-d FEA, as thermally induced stress is a recognized source of bar or joint failure [11,12]. Further, transient conditions such as starting were analyzed to find the worst case thermal distribution for stresses [13]. Results showed that adding the integrated sleeve in the end ring resulted in a gradual distribution of the compressive reaction contact stresses in the soldered joint. The endring without the sleeve resulted in the generation of high compressive and shear stresses in the soldered joint at the inboard section of the endring hole (on the laminations side). The $0.76 \mathrm{~mm}$ thick sleeve reduced the reacting compressive forces on the conductor bar and solder joint. Subsequently, the shear stresses in the joint were reduced. The shear stresses prior the introduction of the sleeve were estimated above $25 \mathrm{ksi}$, but were not quantified due to numerical singularities (sharp corner stresses). The boss feature resulted in the reduction of the shear stresses to $7 \mathrm{ksi}$ as seen in Fig. 4. In addition, the thin web section of the endring reduced the axial support 
stiffness of the bar, thereby reducing the reaction stresses resulting from thermal axial growth of the conductor bar.

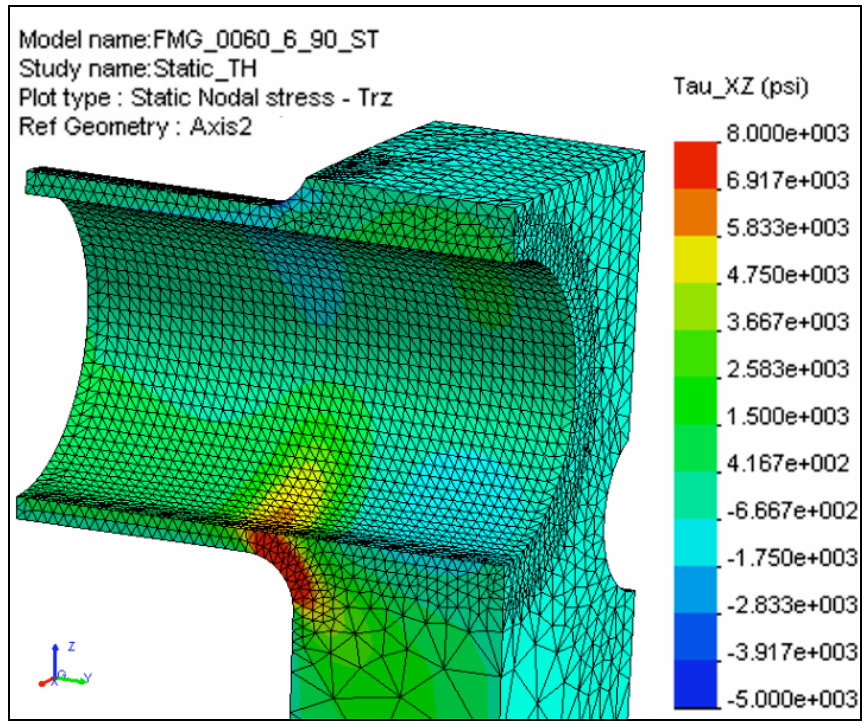

Fig. 4. Shear stresses in joint area are reduced and blended with boss feature

\section{Conical Buckling Prevention Analysis}

Assembly of high-interference fits of laminated cores can result in conical buckling of the structure, thereby partially relaxing the interface preload. In this event there is a possibility of increased radial growth or even axial shifting of the core at speed. Methods of predicting buckling of an unsupported core are currently inaccurate. For highinterference fits, CEM employs a design approach of supporting the core with structure to axially bolster the laminations to contain buckling during all stages of assembly. In this particular induction motor design, the thick section endring is used as a substantial component of the axial bolster structure to prevent conical buckling of this $0.15 \mathrm{~mm}$ radial interference fit.

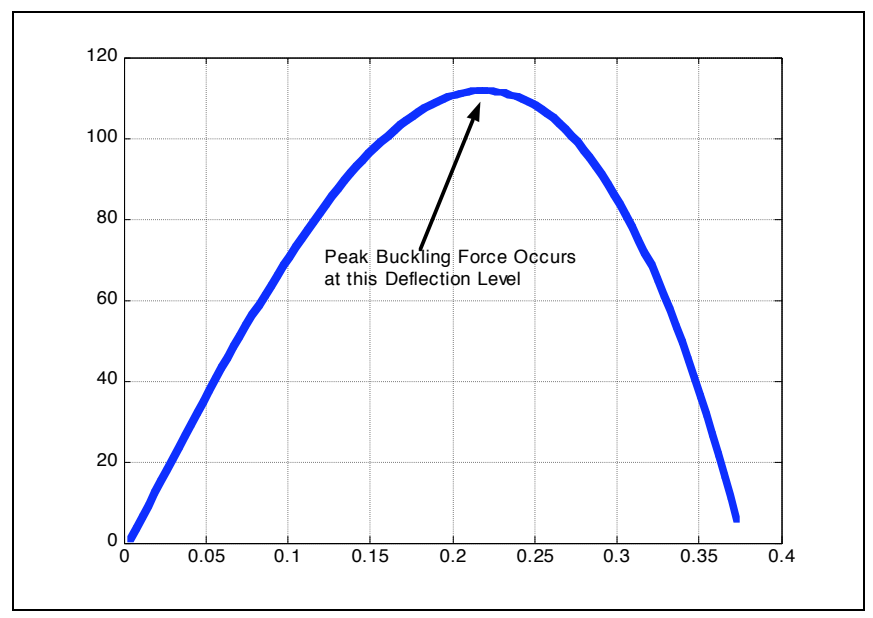

Fig. 5. Axial buckling force of a single 29 gauge lamination
The buckling prevention and containment analysis begins with conservative prediction of the axial load. A closed form expression is used to predict the force-deflection curve (Fig. 5) of a single lamination under conical buckling (this can be confirmed with laboratory testing of a buckled lamination). The gross load of the total number of laminations is then applied to an FEA model (Fig. 6) of the axial containment structure without subtraction of friction. In this case, the peak buckling load is approximately $490 \mathrm{kN}$.

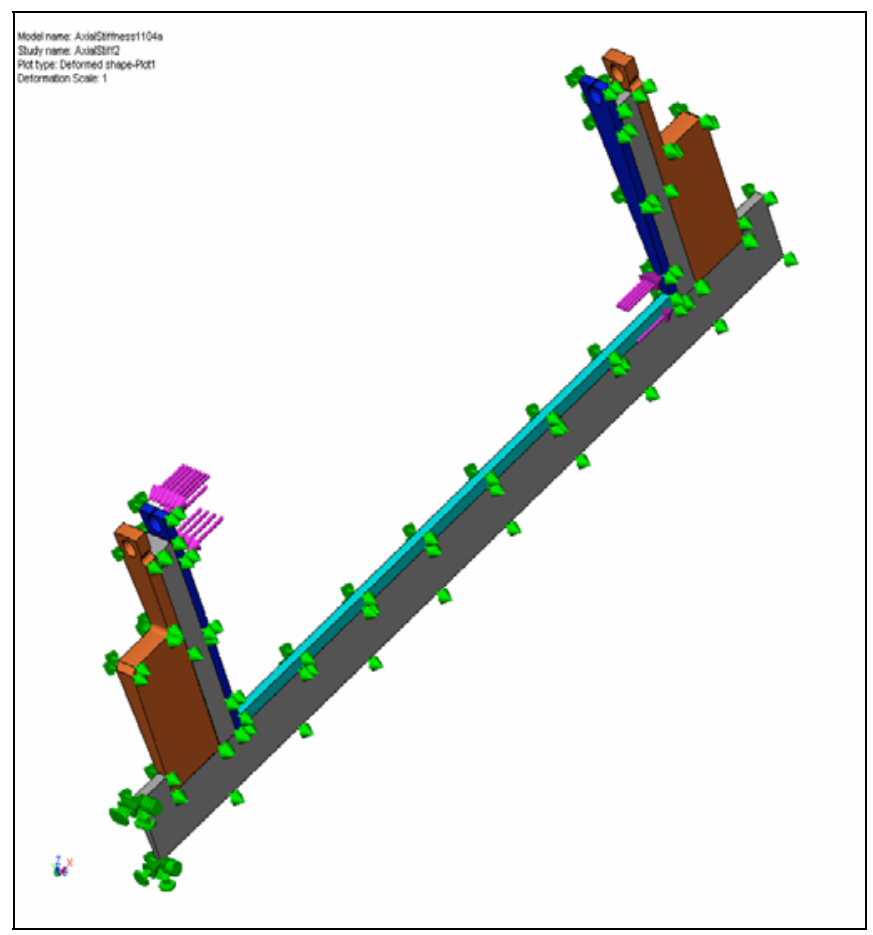

Fig. 6. FEA application of axial buckling loads to support structure

Results of the FEA model indicate approximately 2.3 $\mathrm{mm}$ of deflection result from the conservative buckling load, with a linear stiffness (Fig. 7). If the resulting deflected shape of the structure is less than $50 \%$ of the displacement associated with the peak deflection force, the structure is considered stable against buckling. In this case, the resulting displacement was $42 \%$ of the critical buckling deflection. Due to the parabolic shape of the characteristic forcedeflection curve of the axial load, it will never intersect the linear stiffness of the structure, and therefore essentially zero buckling will occur. This assumption can be assured if the core structure is tightly clamped and axially preloaded with an appropriate fixture during the temperature equalization phase of the shrink fit. If however, free clearance enters the system, an intersection of the two curves is possible, and the resulting amount of contained buckling can be predicted. 


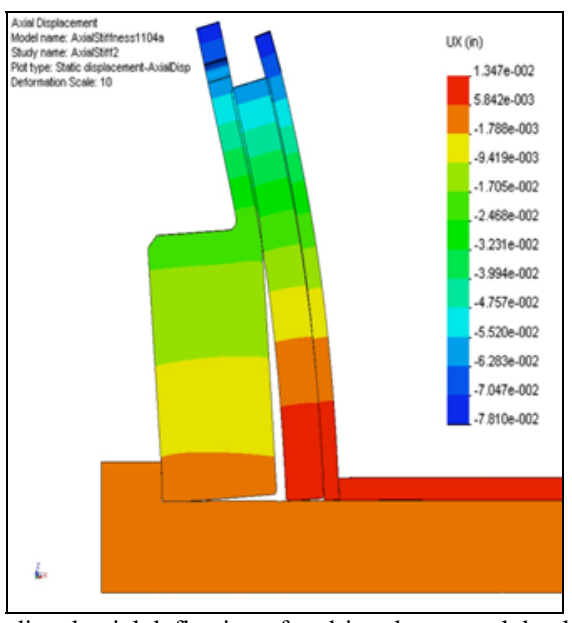

Fig. 7. Predicted axial deflection of endring due to peak buckling load

\section{JoInt StREnGth TeSTING AND PROCEss DEVELOPMENT}

Conventional brazing processes are not feasible for joining the $\mathrm{ZrCu}$ rotor conductor bars to the $\mathrm{BeCu}$ endrings in this application because of the associated strength degradation in the heat affected zone. The high strength (100 ksi) copper alloys achieve their mechanical properties through precipitation age hardening according to specific time and temperature exposure profiles; further processing at elevated temperatures can reduce strength (over aging) or return the material to its soft annealed condition (20-40ksi strength). Brazing of $\mathrm{BeCu}$ requires the use of high copper and zinc content alloys such as RBCuZn-D which necessitate a process temperature of $940-980^{\circ} \mathrm{C}$, which is above the solution annealing temperature range of $900-955^{\circ} \mathrm{C}$. Brazing of $\mathrm{BeCu}$ is therefore followed by re-heat treating of the joined components-- this is impossible with a squirrel cage rotor core assembly constructed of other heat treated components including the steel laminations and shaft. An alternative joining approach is thus required, with process temperatures below the annealing range and preferably below the age hardening temperature $\left(>455^{\circ} \mathrm{C}\right)$ to maintain full strength of the endring and bars.

A low temperature, high strength soldering approach was developed and tested for this application using 80Au20Sn solder typically used by the microelectronics industry for "eutectic die attachment" [14-15]. This solder has a tensile strength rating of $40 \mathrm{ksi}$, and a shear rating of $7.2 \mathrm{ksi}$ at a process temperature of $300^{\circ} \mathrm{C}$. Testing was performed to ensure that adequate joints could be processed with the scale, geometry, and parent metals particular to this application.

A matrix of surface treatments, plating, fluxes, and joint thicknesses was tested to better characterize the achievable shear strength with $80 \mathrm{Au} 20 \mathrm{Sn}$, as there was very little margin between the predicted shear stresses and the rated shear strength of this filler. No measurable improvement in joint strength, wettability, or reflow was measured using electroless nickel immersion gold (ENIG), Sn, Au, or Ni plating for these joints, and ultimately no plating was used.
A process was developed which provided high quality, repeatable joints, and strength tests were performed on actual geometry joints. Shear strengths over $15 \mathrm{ksi}$ (twice the rated strength) were achieved with this filler, resulting in failure of the $\mathrm{ZrCu}$ bar before the joint, proving adequate strength for this application (Fig. 8).

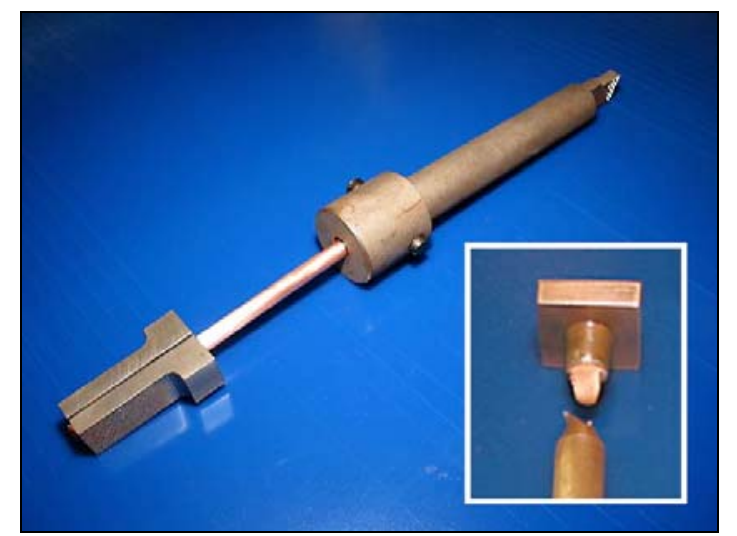

Fig. 8. Solder joint shear test fixture (with inset of failed $\mathrm{ZrCu}$ bar)

An induction heating process was employed to provide a controlled, repeatable heating cycle with reduced risk of localized overheating, and for the purpose of minimizing the oxidation and contamination of the joint surfaces.

\section{PROTOTYPE FABRICATION}

The prototype $2 \mathrm{MW}, 15,000 \mathrm{rpm}$ induction machine employing this design technology is currently in the final assembly stage. The endrings with integrated boss were machined on a CNC mill in order to achieve the high tolerances required for accurate alignment of the 51 rotor bars, given the small solder joint assembly gaps (Fig. 9).

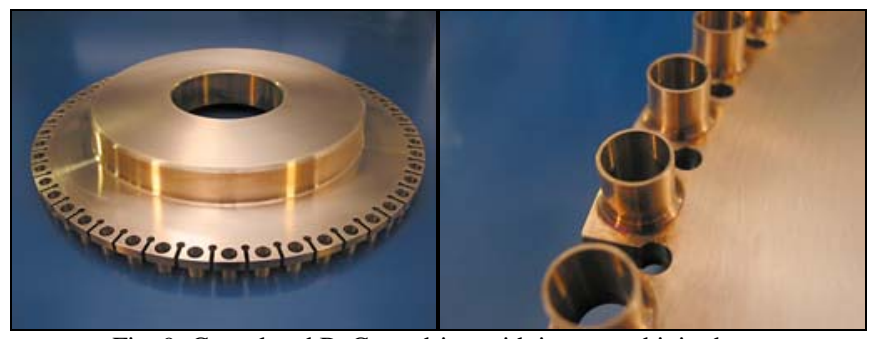

Fig. 9. Completed BeCu endring with integrated joint boss

The interference fit of the rotor core onto the shaft involved heating the core and cooling the shaft to provide adequate geometric clearance for assembly. The core was assembled into a clamping fixture, insulated, and heated with electrical resistance heaters to a uniform temperature of over $290^{\circ} \mathrm{C}$. The shaft was enclosed in dewars and chilled in a liquid nitrogen bath. Upon verification of target temperatures, the dewars was removed, and the core was installed on the shaft and preloaded with a hydraulic cylinder reacting the shaft in tension (Fig. 10). 


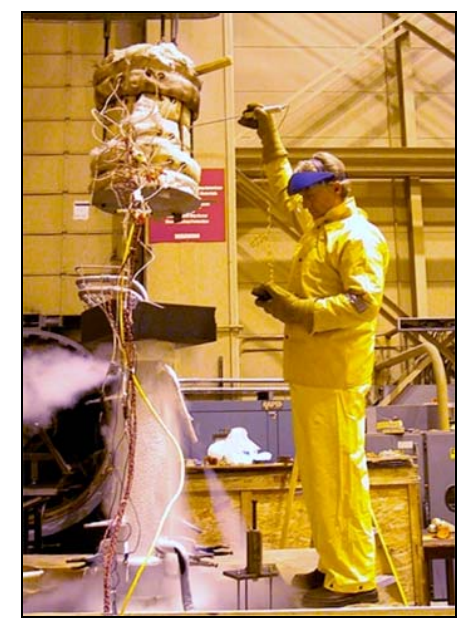

Fig. 10. Assembly of rotor core onto shaft

For the final rotor soldering, a $5 \mathrm{~kW}$ induction heater was used with a custom coil to process each joint individually. Preheating and insulation of the rotor was required in order to prevent heat sinking into the core. Preformed rings of solder were applied to precisely control the amount of filler for each joint. Excess bar material was incorporated into the heating design to provide adequate heat input to the bar during soldering, and then subsequently trimmed. The completed solder joints can be seen in Fig. 11 . The completed rotor (Fig. 12) was then finish machined and balanced for final assembly into the stator.

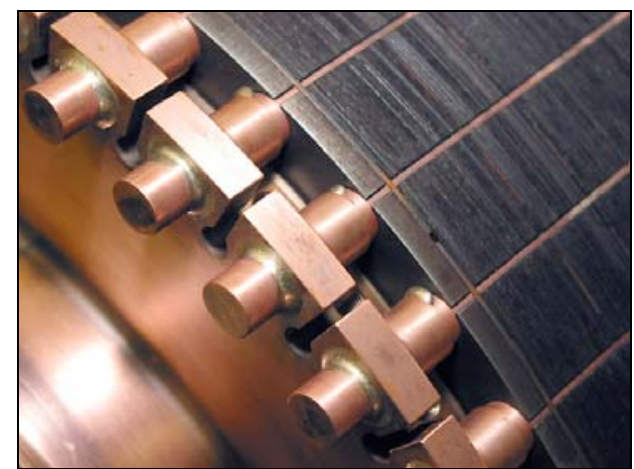

Fig. 11. Completed endring to bar solder joints (before trimming extensions)

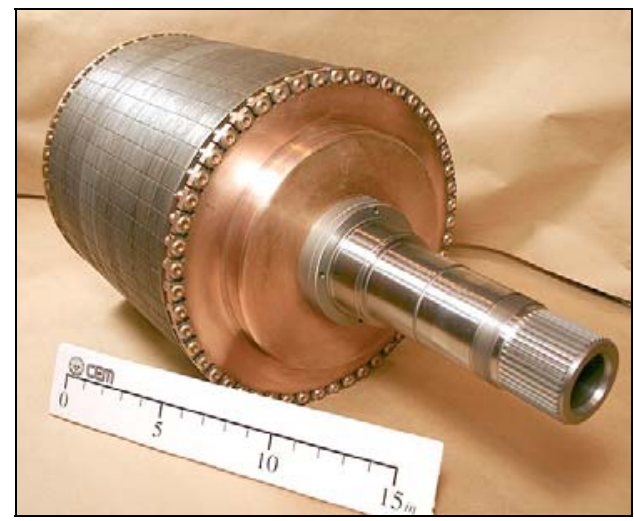

Fig. 12. Completed $2 \mathrm{MW}, 15,000 \mathrm{rpm}$ induction motor rotor

\section{ACKNOWLEDGMENT}

The authors express their gratitude to the Federal Railroad Administration for supporting this work. Additionally, we would like to thank Pratap Singh of Ramp Inc. for his consulting on the development of the soldering specification, joint testing, and processing techniques which made this machine possible.

At CEM, Dr. Howard Jordan must be commended for his vision and oversight of the EM design of this advanced motor generator. Finally, the expert work of technicians Del Beltran and Michael Slotboom is recognized for their attention to detail and creativity in the fabrication and assembly of this machine.

\section{REFERENCES}

[1] A. Bellini, F. Filippetti, G. Franceschini, et al., "On-Field Experience with Online Diagnosis of Large Induction Motors Cage Failures Using MCSA," IEEE Trans. on Industry Applications, vol. 38, no. 4, July/Aug 2002

[2] W.T. Thomson, M. Fenger, "Industrial application of current signature analysis to diagnose faults in 3-phase squirrel cage induction motors", IEEE Conference Record of Annual Pulp and Paper Industry Technical Conference, 2000, p 205-211.

[3] J. Penman, A. Stavrou, "Broken rotor bars: their effect on the transient performance of induction machines", IEE Proceedings: Electric Power Applications, v 143, n 6, Nov 1996 p 449-457.

[4] N. El Kasabgy, A.R. Eastham, G.E. Dawson, "Detection and effects of broken bars in cage rotor induction machines", IEEE Workshop on Electromagnetic Field Computation Proceedings 1987, p G22-G28.

[5] M.T. Caprio, V. Lelos, J.D. Herbst, "Design and Stress Analysis of a High Speed Rotor for an Advanced Induction Motor,” Electric Machine Technology Symposium 2004, American Society of Naval Engineers, January 27-29, 2004, Philadelphia, PA.

[6] J.D. Herbst, M.T. Caprio, R.F. Thelen, "Status of the Advanced Locomotive Propulsion System (ALPS) Project," 2003 ASME International Mechanical Engineering Congress \& Exposition (IMECE '03), November 16-21, 2003, Washington D.C.

[7] M.T. Caprio, J.D. Herbst, R.F. Thelen, "2 MW 130 kWh Flywheel Energy Storage System," Electrical Energy Storage - Applications and Technology (EESAT2003), October 27-29, 2003, San Francisco, CA.

[8] W.R. Finley, M.M. Hodowanec, "Selection of best induction motor rotor construction method", IEEE Conference Record of Annual Pulp and Paper Industry Technical Conference, 2001, p 48-58.

[9] E. Brush, D. Peters, J. Cowie, et al., "Recent Advances in Development of the Copper Motor Rotor," 16th International Conference on Electrical Machines, ICEM 2003,Cracow, Poland, September, 2004.

[10] D. Peters, J. Cowie, E. Brush, et al., "Development of the Copper Motor Rotor- Manufacturing Considerations and Motor Test Results," 6th International Conference on Electrical Rotating Machines, New Delhi, India, February, 2004.

[11] C.H. Jun, A. Nicolas, "Analysis of the mechanical stresses on a squirrel cage induction motor by the finite element method", IEEE Transactions on Magnetics, v 35, n 3 pt 1, May 1999, p 1282-1285.

[12] Y. Liu, H. Jung, S. Hahn, et al., "3D Thermal Stress Analysis of the Rotor of an Induction Motor," IEEE Transactions on Magnetics, v 36, n 4, July, 2000.

[13] M. Pavlicevic, O. Muftic, "Analysis of thermal stresses in rotor of squirrel-cage induction machine", IEEE Transactions on Industry Applications, v IA-23, n 2, 1987, p 334-340.

[14] S. Anhock, H. Oppermann, et al., "Investigations on Au/Sn alloys on different end-metallizations for high temperature applications," IEEE / CPMT Berlin Int'l Electronics Manufacturing Technology Symp, 1998.

[15] C. Kallmayer, H. Oppermann, et al., "Self-Aligning Flip-Chip Assembly Using Eutectic Gold/Tin Solder in Different Atmospheres," IEEE / CPMT Int'l Electronics Manufacturing Technology Symp, 1996. 